\title{
Magnetized Accretion Discs and the Dwarf Nova Phenomenon
}

\author{
P.J. Meintjes*1, E. Breedt ${ }^{1,2}$ and M. Erwee ${ }^{1}$ \\ ${ }^{1}$ Department of Physics, University of the Free State, PO Box 339, Bloemfontein, 9300, South \\ Africa \\ 2 Department of Physics and Astronomy, University of Warwick, UK \\ E-mail: MeintiPJdufs.ac.za / MeintPJegmail.com \\ E.Breedtawarwick.ac.uk, Erweelmats.ac.za
}

In this study dwarf nova eruptions are investigated from the perspective of magnetic viscosity which is parameterized in terms of two quantities, namely the magnetic Reynolds number $\left(R_{\mathrm{m}}\right)$ and the magnetic Hartmann number $(M)$ of the accretion disc. It will be shown that for dwarf novae the disc cycles through low and high states of magnetization. For these systems the rotating magnetosphere outside the co-rotation radius, frozen into the disc, results in an outward centrifugal barrier and an accumulation of the disc, increasing both $R_{\mathrm{m}}$ and $M$. For both $R_{\mathrm{m}}>1$ and $M>1$, magnetic viscosity triggers a magneto-gravitational instability and an accompanying rapid inward advection of the disc plasma with an associated high accretion rate. It is shown that turbulent dissipation in this phase can drive luminosities of the order of $L_{\mathrm{disc}} \leq 10^{35} \mathrm{erg} \mathrm{s}^{-1}$, in accordance with the inferred luminosities during outbursts.

Frontier Research in Astrophysics,

26-31 May 2014

Mondello (Palermo), Italy

\footnotetext{
* Speaker.
} 


\section{Introduction}

Currently it is believed that most outbursts from disc accreting systems like the dwarf nova (DN) phenomenon are intimately linked to significant cyclic changes in the accretion disc viscosity. The quantification of accretion disc viscosity received enormous impetus with the incorporation of turbulent viscosity, which exceeds molecular viscosity by several orders of magnitude. In the seminal paper of [1], the turbulent viscosity is parameterized by $v_{\text {turb }}=\alpha c_{\mathrm{s}} H$, where $\alpha<1$ is a dimensionless parameter which scales the magnitude of the viscosity in terms of the sound speed $c_{\mathrm{s}}$ and the disc scale height $H$. Following this model, the hysteresis S-curve characterizing a DN outburst in the $\Sigma-T_{\text {disc }}$ plane has been quantified in terms of a cyclic $\alpha$ parameter variation ([2]). Simulated DN lightcurves resemble observed lightcurves most closely for values of $\alpha_{\text {cold }} \sim 0.01$ and $\alpha_{\text {hot }} \sim 0.1-0.3$ ([3]). Although the introduction of turbulent viscosity through the $\alpha$ parameter proved to be a useful way of constraining the magnitude range of the disc viscosity, the absence of a detailed theory of turbulence means that it is no more than a neat way of hiding numerous ill-understood underlying principles. It has been shown that magnetized discs are unstable against the magnetorotational instability (MRI) ([4]). For example, Balbus \& Hawley [5], as well as [6] and [7] showed that the strong coupling between the disc plasma and weak disc magnetic fields via the Lorentz force results in the MRI being an effective mechanism for angular momentum transfer in accretion discs. This mechanism also provides a more quantitative description of the viscosity variations between the "hot" and "cold" states of the accretion disc during the DN outbursts.

In this study, a different approach is followed. The effect of magnetic viscosity will be investigated in terms of the magnetic transport through the disc, which is described effectively by two parameters, i.e. the magnetic Hartmann $(M)$ and Reynolds $\left(R_{\mathrm{m}}\right)$ numbers (e.g. [8]). The Hartmann number is the ratio of magnetic stresses to particle viscosity in a magnetized fluid, while the Reynolds number essentially represents the ratio of the convection and diffusion associated with the magnetic field in the fluid. These two numbers are given respectively by

$$
\begin{aligned}
M & =\left(\frac{\sigma B^{2} L^{2}}{\mu c^{2}}\right)^{1 / 2} \\
R_{\mathrm{m}} & =\left(\frac{4 \pi L v \sigma}{c^{2}}\right)
\end{aligned}
$$

where $\sigma, \mu, B, L$ and $v$ represent the fluid electrical conductivity, the coefficient of dynamical viscosity, the magnetic intensity, the length scale of the field and the bulk flow speed respectively. For both the Hartmann and Reynolds numbers exceeding unity, the magnetic field will play an extremely important role in the dynamics of a magnetized fluid, which can have important consequences in astrophysical environments. In this study, the effect of magnetic viscosity will be investigated in disc accreting cataclysmic variables. It will be shown that magnetized discs can naturally explain episodes of transient accretion which are associated with the dwarf nova phenomenon. The paper will be structured as follows: In the next section a brief discussion will be presented of the role magnetic fields will play in slow rotators, followed by a discussion related to magnetic viscosity, a magneto-gravitational instability, turbulent heating, observational evidence for disc turbulence and a conclusion. 


\section{Magnetic Fields and Slow Rotators}

It has been shown (e.g. [9], and references therein) that for slow rotators with magnetic diffusivity a function of radial distance, the associated disc inflow speed can be determined, which is

$$
-v_{\mathrm{R}} \propto\left(\frac{B_{*}^{2} R_{*}^{6}}{M_{*} \dot{M}^{2}}\right)^{13 / 19} \frac{1-\Omega_{*} / \Omega_{\mathrm{K}}}{\eta^{17 / 19} R^{108 / 19}},
$$

where $B_{*}, M_{*}, \dot{M}, \Omega_{*}, \Omega_{\mathrm{K}}$ and $\eta$ represent the polar value of the poloidal magnetic field, the mass of the compact object, the mass accretion rate, the spin angular velocity, the Keplerian angular velocity and the diffusivity of the plasma respectively. It can be seen that for slow rotators material inside the corotation radius satisfies $\Omega_{*} / \Omega_{\mathrm{K}}=P_{\mathrm{K}} / P_{*}<1$, resulting in an inward flow of material, while for regions in the accretion disc outside the corotation radius $\Omega_{*} / \Omega_{\mathrm{K}}=P_{\mathrm{K}} / P_{*}>1$, resulting in an outward magneto-centrifugal push ([10]) which effectively impedes the viscosity-driven radial inflow. The effectivity of the outward magneto-centrifugal push of disc material outside the corotation radius depends on the magnetospheric strength of the compact accreting object, and on the diffusivity of the magnetospheric field of the compact object frozen into the disc plasma. It has been shown ([11]) [Fig 13.6, p.63] that this outward push in disc regions outside the corotation radius results in the inflow speed of a magnetized disc to be significantly less than for a nonmagnetized Keplerian disc. Furthermore, [12] showed that Kelvin-Helmholtz instabilities can be triggered in this outer disc regions where the rotational velocity of the magnetospheric field of the rotating object exceeds the Keplerian velocity of the disc. This can result in magnetic bubbles transferring angular momentum outward through the disc. It is believed that the net centrifugal effect of the faster magnetosphere anchored in the disc and the outward transported Kelvin-Helmholtz magnetized bubbles will result in an accumulation of the disc outside the corotation radius and an associated gradual increase in the disc surface density. The gradual magnetization of the disc through magnetic bubbles and the magnetospheric field that diffuses into the disc, will result in the conditions building-up towards the trigger of a magneto-gravitational instability, which may drive enhanced inward flow of the accumulated disc material and associated high mass accretion. The driving mechanism of this instability will be magnetic viscosity in the disc. A brief discussion will now be presented.

\section{Magnetic Viscosity}

It is believed that frozen-in magnetic fields will have a very significant influence on the dynamical properties of accretion disc plasma. It has been shown (e.g. [8]) that the flow of a conducting fluid across magnetic fields will result in a significantly modified flow profile. For example, [8] showed that for $M>>1$ the fluid rams into a magnetic obstruction too strong to advect, and plasma has to trickle across the magnetic obstruction with the typical $\mathbf{E} \times \mathbf{B}$ drift velocity. For $M<<1$ the magnetic field can be transported with the conducting fluid. This principle has been applied to model the mass transfer flow from magnetized secondary stars, for example in AE Aquarii ([13]) and other magnetic cataclysmic variables ([14]). For example, [13] showed that for Roche lobe mass transfer across a strong magnetic field near the $L_{1}$ region, the flow will essentially be decelerated to such an extent that matter will accumulate behind the magnetic barrier leading to sporadic 
fragmented release of plasma and slingshot prominences. This may be the mechanism behind fragmented mass transfer in many cataclysmic variable systems. A similar process may be operational in accretion discs.

It has been mentioned earlier that the interaction between the rapidly rotating magnetosphere and the slower disc outside the corotation radius of slow-rotators can result in the creation of magnetic bubbles that will transport angular momentum outwards. This will also result in the magnetization of the disc over length scales that comprise a significant fraction of the disc outside the corotation radius. This build-up phase from a situation where $M<1$ (weakly magnetized state of the disc) to a situation where $M>1$ (highly magnetized state of the disc) may define the timescale for the trigger of a magneto-gravitational instability in some classes of accretion driven systems. It will be shown that a magneto-gravitational instability, leading to rapid inflow through the disc and enhanced mass accretion, can be associated with the scenario where $M \geq 1$.

\section{A Magneto-Gravitational Instability}

The build-up of material in a magnetized disc may lead to the disc becoming more turbulent, since the flow outside the corotation radius has departed from a purely Keplerian profile ([11]). It can be shown that in the event of a turbulent disc, where the turbulent disc viscosity exceeds $v_{\mathrm{T}}=\left(\mu_{\mathrm{T}} / \rho_{\text {disc }}\right)=\alpha H_{\text {disc }} c_{s} \geq 10^{13}(\alpha / 0.1)\left(H_{\text {disc }} / 10^{8} \mathrm{~cm}\right)\left(c_{\mathrm{s}} / 10^{6} \mathrm{~cm} \mathrm{~s}^{-1}\right) \mathrm{cm}^{2} \mathrm{~s}^{-1}$ and magnetic diffusivity $\eta_{\mathrm{T}} \sim\left(c^{2} / 4 \pi \sigma_{\mathrm{T}}\right) \geq 10^{13} \mathrm{~cm}^{2} \mathrm{~s}^{-1}$ with frozen-in disc magnetic fields exceeding $B \sim 100$ G,

$$
M>1\left(\frac{B_{\text {disc }}}{100 \mathrm{G}}\right)\left(\frac{L}{H_{\text {disc }}}\right)\left(\frac{v_{\mathrm{T}}}{10^{13} \mathrm{~cm}^{2} \mathrm{~s}^{-1}}\right)^{-1 / 2}\left(\frac{\sigma_{\mathrm{T}}}{10^{7} \mathrm{~s}^{-1}}\right)^{1 / 2} .
$$

With the disc magnetized to this level the gas will increasingly find the disc magnetic fields a barrier that will hamper the azimuthal flow. The braking effect of the azimuthal flow is illustrated by the equation of motion of the azimuthal flow cutting perpendicularly across magnetic fields frozen into the disc, which is

$$
\rho \frac{d \mathbf{v}_{\perp}}{d t}=\mathbf{f}_{\perp}-\frac{\sigma_{\mathrm{T}} B^{2}}{c^{2}}\left(\mathbf{v}_{\perp}-\mathbf{w}\right)
$$

where $\mathbf{f}_{\perp}$ represents the local non-electromagnetic forces influencing the gas flow in the disc and with $\mathbf{v}_{\perp}-\mathbf{w}$ representing the local relative velocity of the disc flow and the frozen-in magnetic fields. Notice that the drift velocity (w) essentially determines the rate at which the magnetic field migrates through the fluid (e.g. [15]). The acceleration experienced by disc plasma will influence the local disc dynamics over an increment of time $(\delta t)$, which is represented by

$$
\begin{aligned}
v_{\phi} & =v_{\phi, i}+\frac{d v_{\perp}}{d t} \delta t \\
& =v_{\phi, i}+\left(\frac{\mathbf{f}_{\perp}}{\rho}-\frac{\sigma B^{2} v_{\phi, i}}{\rho c^{2}}(1-\zeta)\right) \delta t,
\end{aligned}
$$

with $\zeta=\left(w / v_{\phi, i}\right)<1$. One can effectively show that the effective gravity for disc matter orbiting the compact star with mass $\left(M_{1}\right)$ is

$$
\mathbf{g}_{\mathrm{eff}, \perp}=\left(-\frac{G M_{1}}{R^{2}}+\frac{v_{\phi}^{2}}{R}\right) \hat{\mathbf{R}} .
$$


From these equations $\mathbf{g}_{\text {eff }} \rightarrow 0$ for Keplerian flow, i.e. $v_{\phi} \rightarrow v_{\mathrm{K}}$. However, one can see that if the azimuthal flow is decelerated across the fields, $\left.\mathbf{g}_{\text {eff, } \perp<0}<v_{\phi}<v_{\mathrm{K}}\right)$. This means that if the disc has reached critical levels of magnetization, for which the Hartmann number $M>1$, the azimuthal flow will be decelerated such that the effective gravity will point radially inward, which implies that during these states the disc plasma will experience a net inward pull towards the compact accreting object. One can readily show that the effective gravity can be written as

$$
\left.\mathbf{g}_{\mathrm{eff}}=-\left(\frac{2 G M_{1}}{R^{2}}\right)\left(\frac{\sigma B^{2}}{\rho c^{2}}\right)((1-\zeta)-\gamma)\right) \delta t \hat{\mathbf{R}},
$$

where $\gamma=\left(\mathbf{f}_{\perp} / \rho v_{\mathrm{K}}\right) /\left(\sigma B^{2} / \rho c^{2}\right)$. For a turbulent disc with frozen-in magnetic fields of the order $B \sim 100 \mathrm{G}$ and density $\rho_{\text {disc }} \sim 10^{-8} \mathrm{~g} \mathrm{~cm}^{-3}$, it can be shown that $\gamma \sim 10^{-6}$ and $\sigma_{\mathrm{T}} \sim 10^{7} \mathrm{~s}^{-1}$, resulting in

$$
\mathbf{g}_{\mathrm{eff}}=-3000\left(\frac{M_{1}}{M_{\odot}}\right)\left(\frac{R_{\mathrm{disc}}}{3 \times 10^{10} \mathrm{~cm}}\right)^{-2}\left(\frac{\delta t}{1 \mathrm{~s}}\right) \hat{\mathbf{R}} \mathrm{cm} \mathrm{s}^{-2}
$$

which is $\mathbf{g}_{\text {eff }}=3 \mathbf{g}_{\text {earth }}$ for the parameters chosen. It has been observed ([16]) that the most common outbursts are accompanied by an inward propagating heat-wave that originates in the outer parts of the disc, which moves inward with a speed $v_{\mathrm{h}-\mathrm{w}} \sim \alpha_{\mathrm{hot}} c_{\mathrm{s}}$, with $c_{\mathrm{s}} \sim 10^{6}\left(T_{\mathrm{disc}} / 10^{4} \mathrm{~K}\right)^{1 / 2} \mathrm{~cm} \mathrm{~s}^{-1}$ the speed of sound in the hot state of the disc.

\section{Turbulent Dissipation and Heating}

The trigger of the magneto-gravitational instability and associated inward advection of the azimuthal flow $\left(M_{\mathrm{hot}} \geq 1\right)$ of the disc will be associated with large scale hydrodynamic turbulence and associated turbulent dissipation of turbulent mechanical energy. The rate of dissipation of mechanical energy per unit volume of the turbulent eddies (e.g. [15]) is

$$
\dot{u}_{\mathrm{T}}=\rho_{\mathrm{disc}} v_{\mathrm{T}, \mathrm{d}}\left(\frac{v_{\mathrm{rms}, \mathrm{d}}}{l_{\mathrm{d}}}\right)^{2},
$$

with $v_{\mathrm{T}, \mathrm{d}} \approx v_{\mathrm{rms}, \mathrm{d}} l_{\mathrm{d}}$, where $v_{\mathrm{rms}, \mathrm{d}}$ represents the turn-over velocity of turbulent eddies in the dissipative regime, and $l_{\mathrm{d}}$ the smallest length scale of turbulent eddies in the dissipative regime. The dissipative length scales are of the order $l_{\mathrm{d}}=\left(R_{\mathrm{e}} / R_{\mathrm{e}, \text { crit }}\right)^{-3 / 4} H_{\mathrm{d}}$ (e.g. [17], and references therein), with $R_{\mathrm{e}}$ the Reynolds number of the flow, and $R_{\mathrm{e}, \text { crit }} \sim 1000$ the critical value for the trigger of turbulence in the flow (e.g. [18]). Here $H_{\text {disc }}$ represents the maximum length scale of turbulent cells, which is constrained by the vertical disc height. The total disc luminosity in a fraction $(\beta)$ of the disc volume during this "hot" stage can then be estimated as

$$
\begin{aligned}
L_{\mathrm{disc}} & \approx \dot{u}_{\mathrm{T}}(\beta R)^{2} H_{\mathrm{disc}} \\
& \leq 10^{35}\left(\frac{\dot{u}_{\mathrm{T}}}{10^{8} \mathrm{erg} \mathrm{cm}^{-3} \mathrm{~s}^{-1}}\right)\left(\frac{\beta}{0.1}\right)^{2}\left(\frac{R_{\mathrm{disc}}}{3 \times 10^{10} \mathrm{~cm}}\right)^{2}\left(\frac{H_{\mathrm{disc}}}{10^{8} \mathrm{~cm}}\right) \mathrm{erg} \mathrm{s}^{-1} .
\end{aligned}
$$

This upper limit is completely consistent with the observed luminosities of $L_{\mathrm{disc}} \sim 10^{34} \mathrm{erg} \mathrm{s}^{-1}$ of systems like U Gem, SS Cyg, Z Cha and VW Hyi during outbursts (e.g. [19], and references therein). 


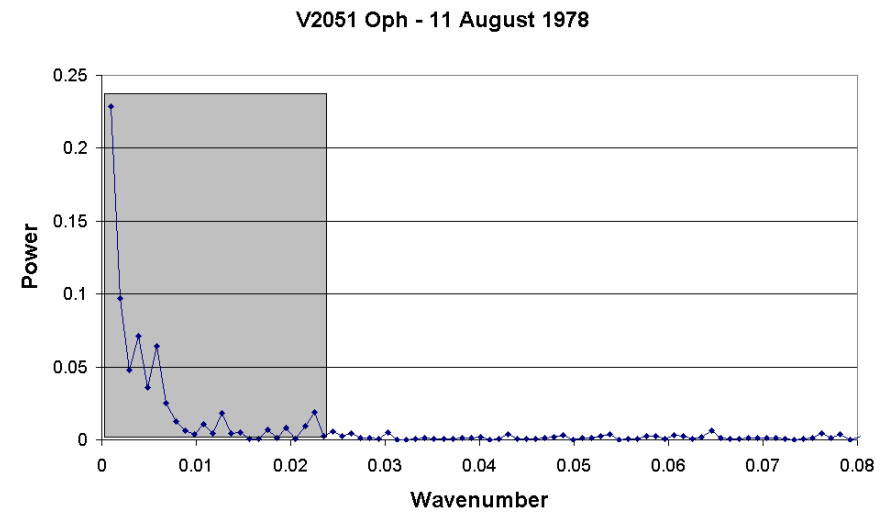

Figure 1: Fast Fourier Transform (FFT) of the flickering lightcurve of V2051 Oph

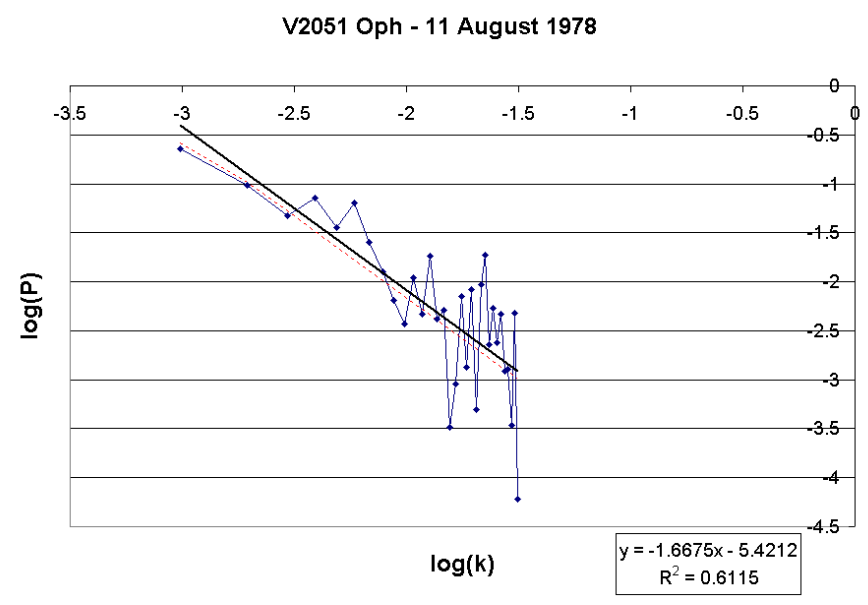

Figure 2: Evidence of a Kolmogorov turbulence spectrum if Fourier power is plotted as a function of wavenumber for the flickering of V2051 Oph

\section{Observational Evidence for Disc Turbulence}

The hydrodynamic stability of a rotating incompressible fluid is determined by Rayleigh's criterion, which states that the flow is stable against linear perturbations if the specific angular momentum increases outward (e.g. [6], [17] for a discussion), given by

$$
\frac{d\left(r^{2} \Omega\right)^{2}}{d r}>0
$$

The condition above is satisfied in any Keplerian flow, where $\Omega \propto r^{-3 / 2}$, which means that a Keplerian flow is stable against linear perturbations (e.g. [6]). However, we showed earlier that magnetized slow rotators can significantly perturb the Keplerian flow profile outside the corotation radius, and cause a breakdown of this stability condition. These discs may be unstable against linear perturbations that may lead to hydrodynamic and magnetohydrodynamic turbulence. The presence of turbulence may be hidden in the flickering of dwarf novae lightcurves ([16]). The flickering 
lightcurves of dwarf novae display a characteristic red-noise behaviour, which can be attributed to the swirling motion of turbulent eddies in the accretion disc. Larger eddies are usually brighter and have a slower swirling velocity than the smaller and subsequently fainter eddies ([16]). Since the turbulent spectrum for the mechanical energy as a function of wavenumber $k$ in the inertial range displays a characteristic Kolmogorov $E(k) \propto k^{-5 / 3}$ law (e.g. [15], [17], and references therein), it is hypothesized that the Fourier power for red-noise should display a similar scaling with respect to wavenumber. To test this hypothesis, several flickering lightcurves of dwarf nova candidates have been tested for periodicity, expressed in terms of wavenumber, over a wide wavenumber range. The smallest wavenumber corresponds to the largest eddies, with sizes constrained by the thickness of the accretion disc, i.e, $l=H_{\text {disc }} \sim 10^{8} \mathrm{~cm}$. The red-noise part of the FFT has been isolated and the Fourier power plotted against wavenumber. These results have been calculated for several sources, but for illustration purposes, displayed graphically only for one particular source, namely V2051 Oph (e.g Fig. 1 and Fig. 2). It can be seen from Fig. 2 that there seem to be a $k \approx 5 / 3$ scaling for Fourier power against wavenumber, as expected for a turbulent accretion disc, with similar linear relations observed from our other dwarf nova candidates.

\section{Discussion}

In this study the effect of frozen-in magnetic fields in the accretion disc has been investigated, expressed in terms of the Hartmann number $(M)$. It has been shown that the accretion discs in slow-rotators may cycle between states of low magnetization $\left(M_{\text {cold }}<<1\right)$ and high magnetization $\left(M_{\text {hot }} \geq 1\right)$, in a similar fashion as the disc cycling between states of low $\left(\alpha_{\text {cold }} \sim 0.01\right)$ and high $\left(\alpha_{\text {hot }} \sim 0.1-0.3\right)$ viscosity during "cold" and "hot" states. It has been shown that during low states of the disc, i.e. low magnetization $(M<1)$, corresponding to low viscosity ( $\alpha \rightarrow \alpha_{\text {cold }}$ ), the frozen-in magnetic field can readily be advected along with the flow, resulting in an effective large scale magnetization mechanism in the disc. It has been shown that in the cold state the centrifugal push of the magnetospheric fields anchored in the disc can result in a low inflow rate through the outer disc, interpreted as a low disc viscosity $\alpha \rightarrow \alpha_{\text {cold. }}$. This will result in a gradual build-up of disc material outside the corotation radius. As the disc grows, the magnetization will increase accordingly, which may lead to the trigger of a large scale magneto-gravitational instability when $M \geq 1$. This leads to an inward-driven mass flow since $\mathbf{g}_{\text {eff }}<0$, which will effectively drain the disc, which will be interpreted as a high effective viscosity $\alpha \rightarrow \alpha_{\text {hot }}$. It has also been shown that dissipation of turbulent energy during this state can easily account for accretion disc luminosities $L_{\mathrm{disc}} \leq 10^{35} \mathrm{erg} \mathrm{s}^{-1}$, which is sufficient to explain the dwarf nova phenomenon.

\section{References}

[1] N.U. Shakura \& R.A. Sunyaev, Black holes in binary systems. Observational appearance, A\&A 24 (1997) 337

[2] F. Meyer \& E. Meyer-Hofmeister, On the Elusive Cause of Cataclysmic Variable Outbursts, A\&A 104 (1981) L10 
[3] A. Ichikawa \& Y. Osaki, Time evolution of the accretion disk radius in a dwarf nova, PASJ 44 (1992) 15

[4] E.P. Velikhov, Stability of an ideally conducting liquid flowing between cylinders rotating in a magnetic field, Sov. Phys. JETP 36 (1959) 1389

[5] S.A. Balbus \& J.F. Hawley, A powerful local shear instability in weakly magnetized disks. I - Linear analysis. II - Nonlinear evolution, ApJ 376 (1991) 214

[6] S.A. Balbus \& J.F. Hawley, Instability, turbulence, and enhanced transport in accretion disks, Rev. Mod. Phys. 70 (1998) 1-73

[7] S.A. Balbus, Enhanced Angular Momentum Transport in Accretion Disks, ARA\&A 41 (2003) 555

[8] J.D. Jackson, Classical Electrodynamics (2nd ed), Wiley \& Sons, New York, (1975) 473, 477-479

[9] C.G. Campbell, Magnetohydrodynamics in Binary Stars, Kluwer Academic Publishers (Dordrecht Boston London), (1997) 171-191

[10] J. Li \& D.T. Wickramasinghe, On spin-up/spin-down torque reversals in disc accreting pulsars , MNRAS 300 (1998) 1015

[11] E. Breedt, A magnetohydrodynamic perspective on magnetic viscosity and mass transfer in disc-accreting cataclysmic variable stars, M.Sc thesis, University of the Free State (UFS), Unpublished, (2005) 63

[12] Y-M. Wang \& J.A. Robertson, A numerical investigation of the Kelvin-Helmholtz instability in the context of accreting neutron stars, A\&A 139 (1984) 93

[13] P.J. Meintjes, Magnetized fragmented mass transfer in cataclysmic variables: AE Aquarii, a trial case, MNRAS 352 (2004) 416

[14] P.J. Meintjes \& E. Jurua, Secondary star magnetic fields in close binaries, MNRAS 372 (2006) 1279

[15] A.R. Choudhuri, The physics of fluids and plasmas, Cambridge Univ. Press., Cambridge, (1998) 260-262

[16] C. Hellier, Cataclysmic Variable Stars, How and why do they vary, Springer and Praxis Publishing, Chichester, (2001) 70-71

[17] D. Biskamp, Magnetohydrodynamic Turbulence, Cambridge Univ. Press., Cambridge (2003) 178-181

[18] D.J. Tritton, Physical Fluid Dynamics, van Nostrand Reinhold, (1977) 233-237

[19] B. Warner, Cataclysmic Variable Stars, Cambridge Univ. Press., Cambridge (1995) 214 